\section{Tres ejes de diálogo epistemológico para aproximarse a una interpretación de la relación ser humano- naturaleza}

Three axes of epistemological dialog to approach an interpretation of the human-nature relationship

\section{Maria Luisa Eschenhagen ${ }^{*}$}

\section{Resumen}

El presente texto es el resultado de una reflexión teórica, después de mi participación en dos proyectos interdisciplinarios de investigación, que visibilizaron claramente las grandes dificultades de este tipo de trabajo y del entendimiento mutuo. Este texto propone lo que podría ser un marco teórico para comprender la

Profesora investigadora de la Escuela de Ciencias Sociales de la Universidad Pontificia Bolivariana, sede Medellín, Colombia, coordinadora del Grupo Territorio. Correo electrónico: mariesche22@yahoo.com.mx. relación ser humano-naturaleza y, con éste, los problemas ambientales. Para tal fin se propone primero una reflexión sobre la importancia de una fundamentación epistemológica en un proyecto de investigación para luego proponer tres ejes de lectura frente a una situación específica, como p.ej. un problema ambiental. El primer eje es la lectura macro con marcos filosóficos, el segundo, el juego de poderes con influencia sobre el entorno y el tercero, las bases materiales sobre las cuales se da la relación ser humano-naturaleza. Se hará un mayor énfasis en el primer eje, ya que tiene una influencia significativa sobre los otros dos.

Palabras claves: pensamiento ambiental, investigación interdisciplinaria, problemas ambientales, relación ser humano- naturaleza.

\begin{abstract}
The present text is the result of a theoretical reflection, after my participation in two interdisciplinary research projects, which showed the difficulties of doing this kind of work and of mutual understanding. This text proposes what could be a theoretical framework for the understanding of the human-nature relationship and, with this, environmental problems. For that aim, I suggest first a reflection on the importance of an epistemological ground in a research project and then I propose three levels of lecture for a specific situation, i.e. an environmental problem. The first axe is the macro lecture with philosophical frameworks; the second, is the game of power which has influences over the environment; and the third, the material bases for the human-nature relationship. We will focus mainly on the first
\end{abstract}


axe, because it has significant influence on the other two.

Key words: environmental thought, interdisciplinary research, environmental problems, human-nature relationship.

\section{A manera de introducción}

Antes de iniciar el texto propiamente dicho, resulta importante hacer una contextualización, en aras de explicitar el contexto que posibilitó las reflexiones teóricas aquí presentes. Ni reflexiones ni resultados hacen parte de un proyecto específico de investigación, sino más bien emergen después de haber participado en dos proyectos de investigación que tenían en común la preocupación ambiental y el autodenominarse interdisciplinarios, así como el de contar con más de 10 investigadores que provenían en un 90\% de las ciencias duras y aplicadas ${ }^{1}$. Otro aspecto en común fue que ambos proyectos fueron concebidos de manera similar, en el sentido de ser escritos y propuestos sin la participación, discusión, ni concesión del respectivo grupo de investigadores; estos más bien fueron contratados y comprometidos a la causa a posteriori.

Proyecto realizado en la Universidad Nacional de Colombia, a través del Instituto de Estudios ambientales, titulado: "Caracterización de los procesos de apropiación y transformación del espacio geográfico con destino a la producción agropecuaria y diseño de la valoración económica parcial ambiental en Páramo de Guerrero (Cundinamarca)", en el 2008. Y una investigación enmarcada en el proyecto del Sistema General de Regalías, de Colombia, denominado: Estrategias de apropiación y valoración de los recursos naturales como mecanismos de adaptación al cambio climático, región del Bajo y Medio Magdalena Cundinamarca, financiado por la Gobernación de Cundinamarca y ejecutado por el Centro Internacional de Física y el Grupo Territorio de la Universidad Pontificia Bolivariana entre los años de 2014 y 2015. En el proyecto de la Universidad Nacional participé como asesora, asistiendo a las reuniones de socialización y discusión de los avances de la investigación, y en el segundo como coordinadora del proyecto, procurando lograr una coherencia entre los lineamientos planteados desde el Centro Internacional de Física quien tenía el liderazgo central, los resultados de campo y los marcos teóricos.
Me llamó la atención que hubo una similitud en las discusiones y dificultades de lograr acuerdos, entendimientos y consensos mínimos entre los investigadores provenientes de las ciencias sociales y de las ciencias duras y aplicadas, donde las ciencias sociales siempre tenían mayores dificultades de ser escuchadas y respetadas (si no querían ser simplemente instrumentalizadas paraimplementartecnologías en las comunidades locales). Se visibilizaron también problemas de comunicación, en el sentido de tener que especificar, discutir y concretar definiciones conceptuales mínimas para lograr un lenguaje común, porque por supuesto cada disciplina tiene sus propios lenguajes, metodologías y códigos, así como concepciones de objetividad, cientificidad, importancia/lugar de la epistemología, etc. Los problemas entre las llamadas dos culturas quedaron evidenciadas, a pesar de tener un cierto acuerdo mínimo y una preocupación en común, de que los problemas ambientales solo se pueden trabajar interdisciplinariamente, al estar implicada la propia relación ser humanonaturaleza. Sin embargo, esto resultó en ambos casos casi imposible, en el sentido estricto de la interdisciplinariedad, y se dieron más bien trabajos multidisciplinarios, que necesariamente terminaron siendo parcialmente incoherentes en sus marcos teóricos y epistemológicos.

Sin desconocer en absoluto las largas y voluminosas discusiones que existen al respecto, señalo para ello a manera de ejemplo a Boaventura de Souza Santos (2004), su excelente libro titulado Conhecimento prudente para uma vida decente. Um discurso sobre as ciências, donde el título ya habla por sí solo. El objetivo aquí definitivamente no es profundizar en estas discusiones, ni tampoco explorar las discusiones y propuestas que existen 
para superar la divisoria relación sociedad/ naturaleza, característica de occidente, con autores como Bruno Latour, Arturo Escobar, Donna Haraway o Mario Blazer, o con metodologías de Silvia Rivera Cusicanqui o Gloria Anzaldúa, que efectivamente son muy interesantes y sugerentes. Se trata, más bien, observando el problema y la dificultad concreta en este tipo de proyectos de investigación, de llamar la atención sobre la necesidad de escribir y discutir conjuntamente entre todos los investigadores participantes el proyecto mismo de investigación, y para ello ofrecer aquí un mapa de navegación argumentado que posibilite, frente al reto, comprender los problemas ambientales complejos a partir de la interpretación de la relación ser humanonaturaleza, para plantear una propuesta de proyecto coherente.

Toda medición modifica la realidad en el intento de registrarla.

Toda conceptualización se basa en compromisos filosóficos.

Con el tiempo, la creencia generalizada en una neutralidad ficticia ha pasado a ser un obstáculo importante al aumento del valor de verdad de nuestros descubrimientos. (Wallerstein, 2001: 82)

Este epígrafe de Wallerstein, tan acertado, muestra muy bien el dilema y problema que significa plantear un marco teórico. El marco teórico de una investigación puede ser o lo más fácil y rápido por definir, si se toman y retoman marcos ya establecidos, o lo más complejo y difícil de establecer. En muchas investigaciones, el marco teórico no es cuestionado a profundidad, ya que las expectativas desde un comienzo están dirigidas a resultados claros que solamente se desean verificar o ajustar a parámetros "aceptables", ya sea para consultorías o para ciertas revistas. Si el marco no es cuestionado, es relativamente fácil y rápido establecer los marcos conocidos y ya aceptados por la mayoría de la comunidad científica (Kuhn, 2000), y comenzar de una vez aplicando el marco teórico y las respectivas metodologías que se asocian con él al objeto a investigar. Esto se puede observar en muchas investigaciones en torno al ambiente, en las cuales el objetivo es encontrar las "mejores" estrategias para su conservación y/o gestión, aplicar instrumentos ya desarrollados, sin revisar y/o cuestionar sus supuestos y bases epistemológicas, así como contextos e intereses políticos, económicos, sociales y culturales a largo plazo, de los cuales hacen parte (aunque algunos dirían que significa "alargar" o "demorar innecesariamente el tiempo valioso $(\$)$ ".

Otro es el caso en el que el marco teórico se presenta desde una perspectiva crítica y reflexiva, lo cual plantea retos importantes y complejiza de manera interesante la labor investigativa. Decidir andar por este camino significa asumir distintas dificultades y cuestionar, y suponer, que las aproximaciones tradicionales para entender, analizar, y consiguientemente, proponer soluciones en torno a los problemas ambientales no resulta ni suficiente, ni satisfactorio. Este texto tiene como objetivo proponer un marco teórico, que en este caso gira en torno a la interpretación de la relación ser humano-naturaleza, a partir del cual se puedan entender mejor los problemas ambientales.

Una de las premisas para proponer este camino reflexivo, desde el uso del marco teórico, es 
plantear que el problema ambiental ${ }^{2}$ es una expresión más de la crisis civilizatoria de Occidente, originada por sus formas de conocer con las que se apropia de su entorno natural (Leff 2004; 2006; 2007). La cosmovisión dominante en Occidente sigue siendo la judeocristiana moderna, marcada por una fragmentación y especialización del conocimiento que requiere de una simplificación y homogenización para poder predecir y planificar. A la vez, esta cosmovisión está regida por una sobredeterminación por parte de la racionalidad economicista e instrumental. Todas estas características, en su conjunto, conllevan una forma muy específica de apropiación, es decir, decolonización, explotación y uso del entorno natural: un conocimiento con el cual resulta casi imposible comprender la complejidad ambiental. El presente trabajo pretende evidenciar este problema, así como abrir unas primeras aproximaciones para varios caminos de comprensión.

Resulta importante abrir este espacio de reflexión, ya que, por un lado, en el quehacer diario de la investigación en las ciencias duras y aplicadas no hay muchos investigadores ni la oportunidad de reflexionar sobre la "neutralidad" u "objetividad" de las teorías e instrumentos que utilizan. Por otro lado, queda también la inquietud de cuánto espacio reflexivo existe

\footnotetext{
Aquí resulta útil intentar aproximarse a diferentes definiciones de qué se puede entender como un problema ambiental, ya que por lo general es difícil encontrar definiciones al respecto. Según Leff un problema ambiental se entiende como "el efecto que produce la racionalidad formal, instrumental y económica como formas de conocimiento y en su voluntad de dominación, control, eficacia y economización del mundo" (Leff, 2004: 21). Sejenovich plantea que "la problemática ambiental surge cuando una formación económica y social transforma la naturaleza con el fin de elevar la calidad de vida de la población. Actualmente, la sociedad realiza este proceso según la racionalidad económica prevaleciente: la que privilegia el corto plazo y el beneficio privado y que, en muchos casos, incumple con la legislación ambiental y genera una contradicción entre los costos privados y sociales que se expresa tanto en el deterioro de la naturaleza como en la insatisfacción de las necesidades esenciales". (s.f.: 1)
}

en la formación básica de estos profesionales en las universidades. Es decir, espacios para comprender su quehacer en un contexto filosófico, histórico y social. Sin embargo, estos contextos por lo general son considerados reflexiones propias de las ciencias sociales, especialmente cuando en un espacio de trabajo interdisciplinario no pueden ser obviadas.

Una forma de evidenciar este problema, profundamente filosófico y epistemológico, es presentar una gráfica, con el peligro de simplificarlo demasiado y errar en su representación, pues una gráfica es apenas la representación de una idea y no refleja la complejidad que se teje también entre las diferentes ideologías, fundamentos, teorías e instrumentos, donde existen también interrelaciones y retroalimentaciones, así como un sinfín de ramificaciones ${ }^{3}$. Lo que en primera instancia se quiere mostrar es que el proceso de definir y escoger instrumentos no es un asunto ni neutral ni objetivo (ver también Lizcano 2006), ya que cada uno tiene sus bases y su justificación en teorías específicas y no se da de manera lineal ni unívoca, como tal vez pueda parecer a primera vista en la siguiente gráfica 1. También es de señalar, que la gráfica perfectamente se podría plantear y leer de manera inversa.

En esta gráfica se intenta mostrar cómo los instrumentos para solucionar un problema son el producto 0 , valga la redundancia, son la instrumentalización de teorías específicas. Para ilustrar este planteamiento se pueden ver los siguientes ejemplos: tanto el capitalismo

\footnotetext{
Es de señalar aquí que existe una bibliografía muy amplia y rica, y de vieja data, ya sea desde la sociología de la ciencia, los estudios sociales de ciencia y tecnología o desde la propia filosofía de la ciencia que no se puede obviar, pero que claramente se sale y sobrepasa al objetivo del presente trabajo.
} 


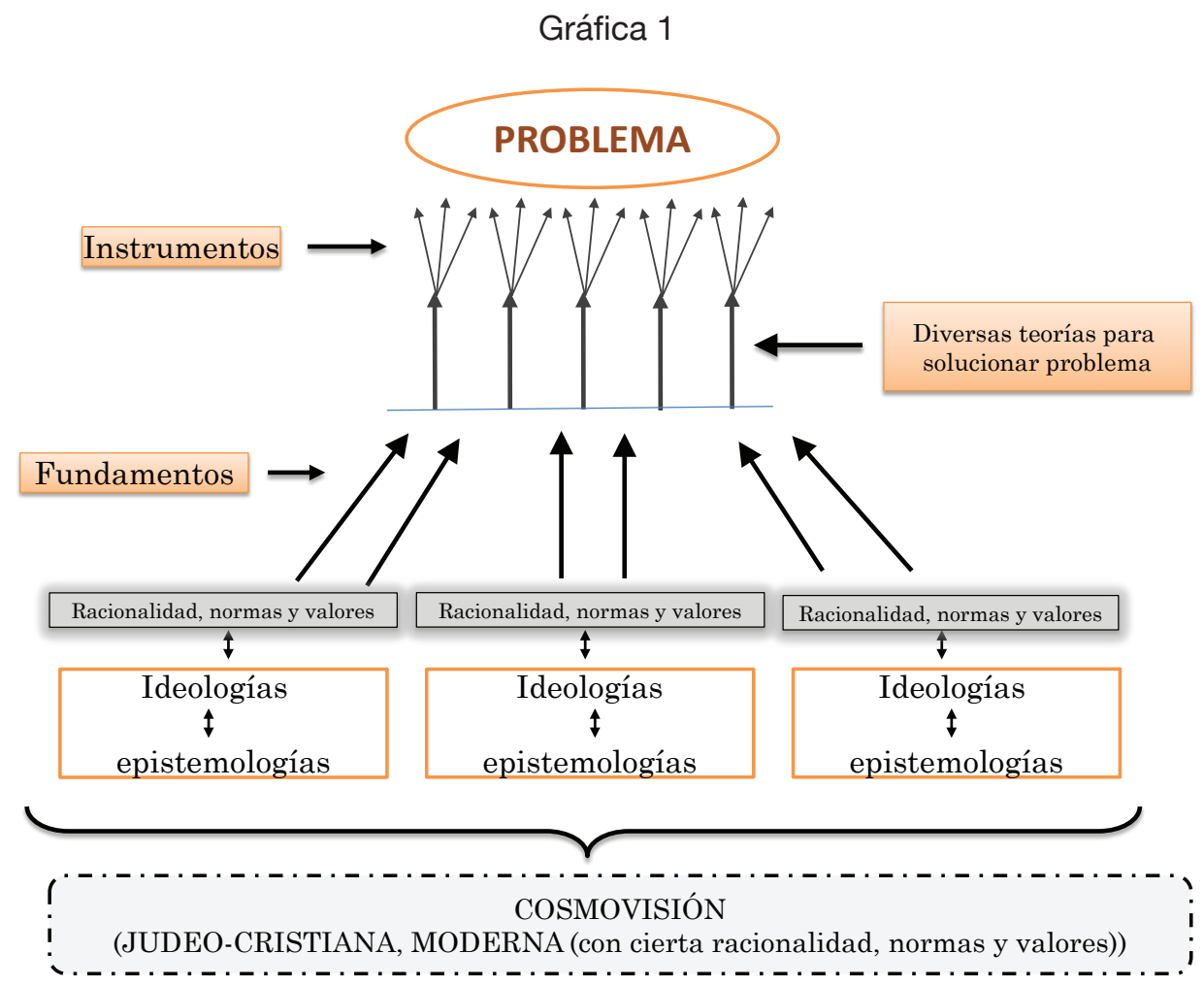

Elaborada por María Luisa Eschenhagen

como el socialismo surgieron de la modernidad, ambos están acompañados por sus racionalidades, valores, normas y formas de construir y validar conocimiento, pero así como tienen sus diferencias, también comparten una cosmovisión, la judeo-cristiana, y ambos terminaron por instrumentalizar y objetivizar la naturaleza para explotarla en función de la industrialización. Otros ejemplos pueden ser la teoría de sistemas y la complejidad como formas específicas de construir conocimiento que a su vez generan diferentes propuestas e instrumentos, y las teorías como el desarrollo sostenible o el ecodesarrollo, que nacieron de ideologías diferentes. Cabe recordar que cada vertienteepistemológicaha desarrolladotambién sus propias metodologías de investigación y por ende serán también diferentes las "soluciones" y proyectos que recomiendan e implementan. Identificar estas diferencias, reconocer los orígenes e implicaciones a largo plazo resulta indispensable, ya que aunque aparentemente se desea llegar a una misma meta, la implementación de los diferentes instrumentos (sin mayor fundamentación teórica explícita) puede tener consecuencias diametralmente opuestas o inducir efectos y procesos que son contradictorios con los fines de cada teoría. 
Aquí también resulta indispensable señalar, por un lado, que en la medida en que el investigador adquiere conocimiento y reconoce las respectivas implicaciones a largo plazo, adquiere también una responsabilidad ética con el conocimiento; y, por el otro, que todas las teorías están atravesadas por intereses de poder socio-políticos y económicos. Desde este contexto, el marco teórico adquiere una importancia significativa dentro de una investigación y sus bases epistemológicas deberían ser evidenciadas y coherentes. Diferenciar y reconocer los matices e intereses que se juegan en los múltiples planteamientos que existen para abordar los problemas ambientales resulta una tarea fundamental del investigador en cualquier disciplina.

Ahora, para llegar a este punto de partida y explicitarlo es necesario realizar unas reflexiones filosóficas, aclaraciones epistémicas, y por ende aclaraciones teóricas, ya que un marco teórico no nace de la nada, sino que está enmarcado dentro de una visión de mundo y se sustenta en unas epistemes e ideologías específicas. Éstas a su vez están contextualizadas en un lugar de enunciación, en un momento histórico, social, económico y político que marca, direcciona, condiciona y posibilita las diferentes teorías ${ }^{4}$. De ahí que los marcos teóricos, y los instrumentos que se proponen, no pueden ser neutrales ni objetivos, ya que al escoger uno también se escoge una posición filosófica, ética y política, y por ende se asume también una responsabilidad. Tomar una postura supuestamente imparcial, al suponer que la ciencia es "neutral" y "objetiva" puede resultar ser cómodo y conveniente

Para profundizar y sustentar esta línea argumentativa, ver trabajos y reflexiones realizados en torno a la colonialidad del saber y la geopolítica del conocimiento, así como del poder en el saber: Galcerán Huguetc (2007), Walsh (2002 y 2003), Lander (2000). pues no es necesario tener que reflexionar mayormente sobre el marco teórico, ni sobre sus implicaciones. La necesidad de escoger un marco teórico y explicitar que está enmarcado en una epistemología e ideología específica no siempre queda claro ni evidente y es de suma dificultad en grupos de investigación con integrantes multidisciplinarios. Por ejemplo, si entre las disciplinas de las ciencias sociales puede haber diferencias y dificultades de comunicación sustanciales, el reto se vuelve aún mayor si estas tienen que entrar en diálogo con las ciencias denominadas duras y aplicadas.

Para este ejercicio se requiere de una gran apertura y madurez, ya que la comunicación, desde un comienzo, cuenta con un sinfín de barreras, pues no se cuenta con un lenguaje en común. Así, por ejemplo, para las ciencias duras y aplicadas un concepto por lo general tiene una definición unívoca, mientras que para las ciencias sociales existen un sinfín de definiciones y los conceptos tienen genealogías y hacen parte de discursos ${ }^{5}$. Por lo tanto, se trata de un proceso arduo y hasta doloroso, que requiere de tiempo (que desafortunadamente, en la mayoría de los casos, en los proyectos financiados no se tiene) y paciencia. De ahí que se podrá hablar mucho teóricamente de interdisciplinariedad ${ }^{6}$, pero la primera barrera a derribar es la humana, que sin confianza, respeto y una disposición constante de diálogo horizontal, no funciona; es decir, se requiere de empatía ${ }^{7}$.

P.ej. qué significa caracterizar, recurso, poder, etc. A manera de anécdota y ejemplo, recuerdo que un químico quería despachar el concepto de poder con la definición que da la Real Academia Española, lo cual definitivamente no es posible hacer en ciencias sociales.

$6 \quad$ Ver la bibliografía que existe al respectos como p.ej.: Aguiar Coimbra (2000), Apostel (1979), Thompson Klein (1990)

7 Ver el trabajo interesante que ha escrito Jeremy Rifkin (2010) al respecto. 
Dentro de esta línea de argumentación, el presente texto se propone, para el análisis de la interpretación de la relación ser humanonaturaleza, plantear tres ejes: primero, la importancia de una lectura macro que da las bases filosóficas y teóricas en lainterpretación de esta relación; segundo, evidenciar la necesidad de considerar el juego de poderes que confluyen sobre el entorno en el cual se manifiesta esta relación, para finalmente señalar la importancia de las bases materiales en las cuales tiene lugar esta relación y que también influyen sobre los otros ejes. Estos tres ejes deberían entonces hacer parte de una propuesta para la interpretación de dicha relación y para entender las dinámicas, trayectorias y transformaciones socioambientales de un lugar específico.

Para visualizar la aplicación de estos tres ejes se proponen las siguientes gráficas. La Gráfica 2 muestra de frente los niveles en que se encuentran los diferentes ejes:
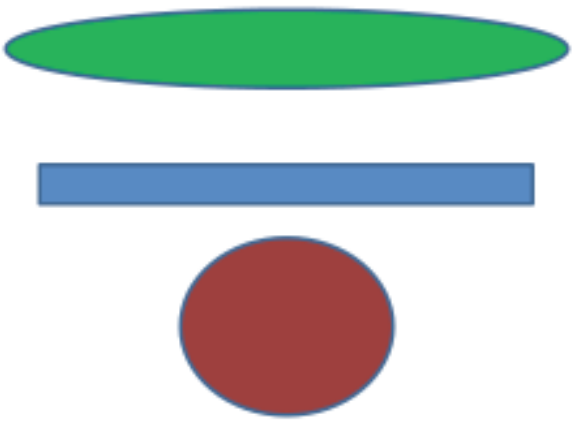

Eje 1 Lectura macro

Eje 2 Juego de poderes con Influencia sobre el entorno

Eje 3 Bases materiales

PROBELMA
Esta es una gráfica dinámica cuyo primer eje está constituido por dos elipses que giran y se pueden concebir a manera de lentes. Es decir, la mirada macro es el marco filosófico/teórico que determinará en buena manera de qué forma se leen, por ejemplo, la política, la economía y las concepciones de poder, así como la elección de los instrumentos y las interpretaciones de los aspectos culturales, tecnológicos o biofísicos. Es de aclarar, además, que una investigación necesariamente tendrá que elegir uno de los dos lentes para mantener la coherencia interna.
Entonces, como se puede ver en la Gráfica 3, en el centro está un problema $X$, en este caso el objetivo de comprender la relación ser humanonaturaleza, y puede ser visto a través de dos lentes.

Ahora, para comprender estos dos lentes resulta indispensable acercarse primero a algunos aspectos de la modernidad que configuran la cosmovisión occidental hegemónica, pues aquello puede dar luces para entender el mundo en el que se encuentran la gran mayoría de las 
Gráfica 3

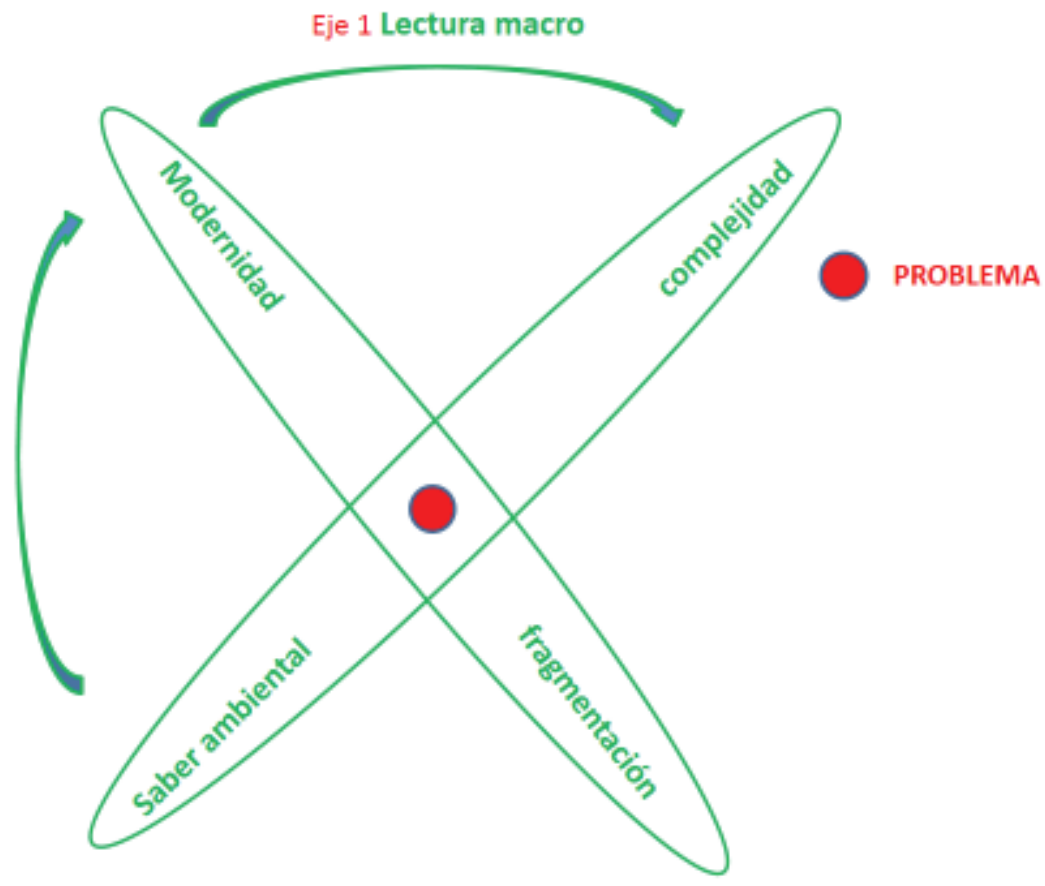

sociedades actualmente. Es decir, entender las convicciones, valores y visiones de mundo que rigen el comportamiento y las acciones facilita plantear las preguntas necesarias para entender aún mejor la visión de mundo hegemónica. Preguntas que de manera consecuente cuestionan críticamente los supuestos, sentidos y consecuencias del conocimiento. Por ejemplo, cuestionar y entender la relación ser humano-naturaleza resulta profundamente filosófico, ya que se juegan preguntas en torno a: qué es el ser humano; qué lo diferencia del animal; si el conocimiento es para habitar o para conocer; o para encontrar la verdad o lo bueno; qué se entiende por naturaleza; si el ser humano depende de la naturaleza o es independiente de ella; en este contexto, qué papel juega la libertad del ser humano; en qué se diferencian los conceptos de naturaleza propuestos por Heráclito, Thomas de Aquino, Newton y Goethe (ver Schiemann 1996 y Ángel Maya 2002); qué aportes han realizado las nuevas ciencias como la complejidad al entendimiento de la naturaleza; qué implicaciones axiológicas y actitudinales tiene cada propuesta para la vida cotidiana; y qué metodologías se desprenden de cada concepto; etc. ${ }^{8}$ La antropología ha demostrado, a través de un sinfín de estudios etnográficos que ha revisado cuidadosamente

Este no es el espacio para profundizar en todas estas preguntas, en otro texto he realizado un panorama justamente sobre las diversas posturas y concepciones de la relación ser humano - naturaleza, sobre diferentes conceptualizaciones de naturaleza y ambiente, que configuran un sinfín de discursos, ver Eschenhagen 2016. 
Descola (2012), las implicaciones ontológicas y epistemológicas que tienen esta diversidad de concepciones sobre las culturas y su habitar del mundo.

El caso de la civilización moderna en este contexto es muy peculiar, ya que a través de la fragmentación del conocimiento que ha propiciado el método científico el entorno natural se convirtió en un objeto, una cosa, que puede ser dominado y explotado. Desconocer o ignorar estas premisas filosóficas básicas, que han forjado la modernidad y por ende las diferentes sociedades, y dar los conceptos de naturaleza y ser humano que se manejan entre los científicos, en los proyectos y la política, por sentados, sin cuestionarlos, significaría limitar, cerrar e invisibilizar aspectos cruciales para entender, en este caso, las interrelaciones ser humano-naturaleza, así como las relaciones de poder que las rigen. Esta visibilización por lo general resulta difícil, ya que esta fragmentación ha sido naturalizada y poco problematizada, especialmente en el "mainstream" de las ciencias duras y aplicadas ${ }^{9}$.

Por lo tanto, como primer paso, es necesario aclarar el lugar de enunciación de cualquier trabajo, es decir especificar y evidenciar a partir de qué supuestos filosóficos y teóricos se construye el marco teórico (y también desde qué lugar geográfico). El punto de partida, y central

\footnotetext{
En las ciencias sociales, especialmente desde la antropología sin embargo existen ya desde hace un tiempo trabajos muy interesantes que intentan romper decididamente estas dicotomías, desde perspectivas como p.ej. las post-humanistas de Haraway (1991 y 1996), las percepciones del ambiente según Ingold (2000), la propuesta de sentipensar con la tierra, y la ontología relacional, de Escobar (2014) Otras aproximaciones, que resultan ser aún más provocadoras, que ya vienen desde los años 70, cuando Thomas Nagel pregunta qué sería ser como un murciélago (Nagel 1974) o más recientemente la propuesta de pensar como un bosque (Kohn 2013) o como un río, según David Brower. Todos estos autores realizan unos esfuerzo grandes e interesantes para superar la visión moderna de naturaleza,
}

desde una perspectiva crítica, es plantear que el problema ambiental es entendido como una expresión más de la crisis civilizatoria por la que está pasando el mundo occidental actualmente. Esto significa que la civilización moderna actual, que ha utilizado un conocimiento muy específico -la ciencia- para apropiarse y transformar el territorio, ya no está en capacidad de ofrecer soluciones satisfactorias a los problemas que crean sus propias estructuras productivas y sociales.

La ciencia moderna se caracteriza por recoger "un interés pragmático, acorde con el intento de dominar la naturaleza" (Mardones 1982: 25) y está marcada por una concepción funcional y mecanicista del mundo, con la pretensión de ejercer poder y control sobre la naturaleza según su racionalidad de utilidad $^{10}$. Desde esta perspectiva moderna, la vida, lo vivo, la naturaleza, es un objeto, una cosa que se puede medir, cuantificar, planificar y por ende explotar y mercantilizar, para ampliar de esta forma la escisión entre ser humano y naturaleza. Perspectiva que aún predomina en la mayoría de los proyectos ambientales de intervención.

La ciencia moderna se aproxima a la naturaleza como a un ente inerte, distante, algo ahí afuera, que nada o poco tiene que ver con los investigadores que trabajan de manera "objetiva" y "rigurosa" ese Otro, ahí afuera. El observador se ubica fuera de la naturaleza y por ende termina por ignorar completamente la conexión con ella ${ }^{11}$. Esta idea se inculca desde

10 Uno de los principales representantes de esta visión es Francis Bacon. Y para ver cómo se da este desencantamiento del mundo, ver el libro de Berman (2001), quien describe muy bien (y de manera muy amena) la revolución científica y sus implicaciones. En esta misma línea está el trabajo de Miranda Vera (1997)

11 Vale la pena aclarar, salvaguardar aquí, que los ecólogos son los que tal vez más claramente reconocen la estrecha interdependencia de la 
el colegio, donde se enseñan las diferentes materias -biología, matemáticas, español, ciencias sociales, etc.- y, por lo general, no se relacionan. Tampoco se fomenta un pensamiento interrelacionado ni interdependiente que sea capaz de comprender la complejidad ambiental. Esta descontextualización se materializa y se expresa finalmente cuando los niños piensan que la leche sale de la nevera, el agua de la llave del baño, y no pueden diferenciar entre lo que es natural y lo que es artificial. También se expresa en que actualmente muchas personas no logran entender que son dependientes del entorno natural, ya que viven "alejados" y se consideran "independientes" de él, al no tener que someterse, por ejemplo, a las horas de luz o a las épocas de cosecha, pues tampoco ayuda que el $70 \%$ de la humanidad vive ya en ciudades $^{12}$. Es dentro de este contexto en el cual Leff ${ }^{13}$ plantea que el problema ambiental resulta ser un problema civilizatorio, que tiene sus raíces en las formas de conocer con las cuales se apropia y transforma el entorno natural de una manera nada sustentable.

También se pueden señalar autores como Maturana y Varela (1998), quienes desde la neurología y biología argumentan que el conocimiento es una forma de adaptación al medio. O un autor como Ángel Maya (1996), quien plantea que la plataforma instrumental que ha creado el ser humano es una forma de

relación ser humano - naturaleza, y la reivindican. Sin embargo, su método científico - propio de las ciencias duras - les impide reconocer, que son hijos de la modernidad. Sería bueno, por lo tanto, que en la formación de los ecólogos se incluyan la antropológica, política, economía, etc., para reconocer los discursos de los que hacen parte.

12 Ver indicadores oficiales del Banco Mundial (http://datos.bancomundial. org/indicador/SP.URB.TOTL.IN.ZS y http://www.bancomundial.org/ temas/cities/datos.htm )

13 Ver gran parte de su obra, en la cual desarrolla este planteamiento: Leff 2000, 2003, 2004, 2006, 2007. adaptación al medio, en la que se encuentran la religión, los mitos, los símbolos, el conocimiento, etc. Desde estas propuestas surge una pregunta: si el conocimiento es una forma de adaptación del ser humano a su entorno natural, ¿qué pasó con ese conocimiento que hoy en vez de adaptarse al entorno natural lo destruye?

Ahora, en torno a este conocimiento moderno, hegemónico que sigue vigente en la gran mayoría de los lugares del mundo moderno, y en un gran porcentaje de la población, en la cual se ha infiltrado y naturalizado este conocimiento, también se observan desde hace más de 40 años, serios cuestionamientos desde el ámbito académico ${ }^{14}$ : críticas en torno a la fragmentación y especialización del conocimiento moderno, que emergen especialmente cuando se trata de comprender la relación ser humanonaturaleza, y con ello la problemática ambiental, cuya complejidad no se puede entender de manera fragmentada.

De ahí se vienen abriendo, buscando y planteando otras formas de conocer, es decir se han cuestionado las bases epistemológicas de la modernidad y buscado caminos para superar la fragmentación del conocimiento a través de la teoría de sistemas, la interdisciplinariedad, y las ciencias de la complejidad, por ejemplo. Cada una de estas tres propuestas tiene alcances diferentes, así como limitaciones. La teoría de sistemas no abandona del todo las bases epistemológicas del positivismo e incluso se podría decir que es un positivismo más sofisticado. Aquí se pueden encontrar las teorías clásicas de Bertalanffy (1968), por ejemplo. Otro asunto son los sistemas

4 En las ciencias sociales esto está muy bien descrito por Wallerstein (2001) en su libro "Abrir las ciencias sociales". 
complejos adaptativos, que ya hacen parte de las ciencias de la complejidad $^{15}$ y un sinfín de aproximaciones a concepciones sistémicas, como bien lo visibiliza Ison (2008). $\mathrm{La}$ interdisciplinariedad tiene una serie de acepciones y, dependiendo de su definición, tendrá alcances diferentes. Mucho se ha escrito en torno a ella, muchos creen realizarla, pero por lo general no logran ir más allá de la multidisciplinariedad ${ }^{16}$. Finalmente, las ciencias de la complejidad, o mejor dicho, las ciencias de la vida, son tal vez el camino más prometedor y a la vez más exigente para superar la fragmentación del conocimiento moderno, ya que exigen por ejemplo distanciarse de la trayectoria mecánica de la ciencia moderna, "que son, ex post, los tres atributos de la humanidad occidental: la legalidad, el determinismo, la reversibilidad... [es decir]... la ciencia moderna es ciencia de intervención, de previsión. La naturaleza será legal, sometida y previsible, y no caótica, irregular, estocástica" (Maldonado 2005: 53,54). Sin embargo, estas exigencias filosóficas y epistemológicas -que a la vez son profundamente éticas-, de pensar en términos de movimiento, imprevisibilidad, irreversibilidad, indeterminación, etc., y que son el resultado consecuente del reconocimiento de la existencia de la entropía y la cuántica, aún no tienen lugar, como ampliamente lo expone el físico cuántico y nobel de paz alternativo Dürr (2009). La dificultad surge también, porque, como dice Maldonado (2013), las ciencias

15 Como bien lo señala Maldonado, "los tres ejes referenciales dentro de los cuales se inscribe el estudio de los sistemas complejos adaptativos (SCA) son los siguientes: a) La comprensión de la complejidad.; b) La actuación sobre la complejidad.; c) El uso, el dominio o el aprovechamiento de la complejidad; [...] los tres ejes deben ser entendidos en sus interrelaciones y co-implicaciones recíprocas" (Maldonado 2003: 141).

16 Para sustentar y demostrar esta afirmación, ver Eschenhagen (2009), donde se realizó una investigación más profunda sobre la interdisciplinariedad, de cómo es entendida y luego asumida y/o aplicada en la formación de maestrías ambientales. de la complejidad son profundamente antiintuitivas ${ }^{17}$.

Otro insumo, para este lente de la complejidad, es el saber ambiental propuesto por Leff, concepto que no puede ser aislado de los otros que propone Leff para un pensamiento ambiental, como la racionalidad ambiental, la epistemología ambiental y el diálogo de saberes. Esto debido a que "la cuestión ambiental es eminentemente política" (Leff s.f.: 4) y pasa por la construcción de conceptos como una estrategia para posibilitar prácticas alternativas. Leff entiende la epistemología ambiental como lo que "da curso a un nuevo saber; un saber que emerge desde la marca de un límite, de una leylímite de la naturaleza, de la ineluctable ley de la entropía" (Leff 2007: 6), y complementariamente como aquella "que es una política del saber que tiene por "fin" dar sustentabilidad a la vida; es un saber para la vida" (Leff 2006: 17). Para lograr esto, Leff propone caminos concretos para develar los obstáculos epistemológicos de la modernidad, en los que priman la racionalidad economicista e instrumental que impiden comprender la complejidad ambiental.

En términos epistemológicos, las ciencias de la complejidad exigen superar también "el lenguaje de la ciencia moderna - posibilitado y determinado al mismo tiempo por la física - [que] es conservativo, a partir de cinco rasgos definitorios de la ciencia moderna:

- se estudian sistemas aislados - o lo que es equivalente, cerrados -, en los cuales la energía se conserva

lo que cuenta es el estado global del sistema de estudio, de suerte que la lógica, la metodología y la gramática de la ciencia moderna son las del formalismo

el concepto de "variables canónicas" representado en la función hamiltoniana $H$, sencillamente expresa y con tiene los cambios reales y posibles, a saber: los cambios "canónicos", por así decirlo; esto es, los únicos cambios posibles, permitidos y reconocidos

- todas las representaciones de un mismo sistema son equivalentes, lo cual significa que la naturaleza se agota en los puntos de vista que engendra. Precisamente por ello un problema central de la ciencia moderna es la elección - discriminación - de los puntos de vista

las interacciones son formalmente suprimidos; los modelos integrables consisten justamente en esto: en la eliminación de interacciones y en la superposición de las trayectorias del sistema" Maldonado, 2005: 56) 
Por lo tanto, el "saber ambiental se forja en el encuentro, enfrentamiento, entrecruzamiento, hibridación y complementación de saberes diferenciados pro matrices de racionalidadidentidad-sentido que responden a estrategias de poder por la apropiación del mundo y la naturaleza" (Leff 2007: 9).

Es dentro de este contexto que una claridad en la fundamentación epistemológica, y por ende filosófica, no puede ser ignorada. A cada lente le corresponde una forma muy específica de construir, posibilitar, justificar, contextualizar y legitimar su conocimiento. No es lo mismo ver y entender la vida, el ser humano o una ciudad desde una cosmovisión moderna o una compleja. Es así entonces como el primer eje representa los lentes, a través de los cuales se mira el problema. Se trata de unos lentes que, por lo general, son naturalizados e incuestionados, pero que determinan significativamente las formas de concebir y comprender la relación ser humano-naturaleza $y$, por ende, los problemas ambientales. Cada lente a la vez influirá en las formas de entender y formular discursos políticos, de identificar y articular actores, así como de plantear y ejecutar tanto teorías como directrices políticas y económicas. Por lo tanto, como bien lo señala Wallerstein: "toda conceptualización se basa en compromisos filosóficos... [y] ... toda medición modifica la realidad en el intento de registrarla" (2001: 82), con lo cual también está implícita ineludiblemente una responsabilidad ética en el manejo del conocimiento, del saber y sus respectivas capacidades y consecuencias de transformación del entorno.

El segundo eje se puede representar de la siguiente manera:

\section{Gráfica 4}

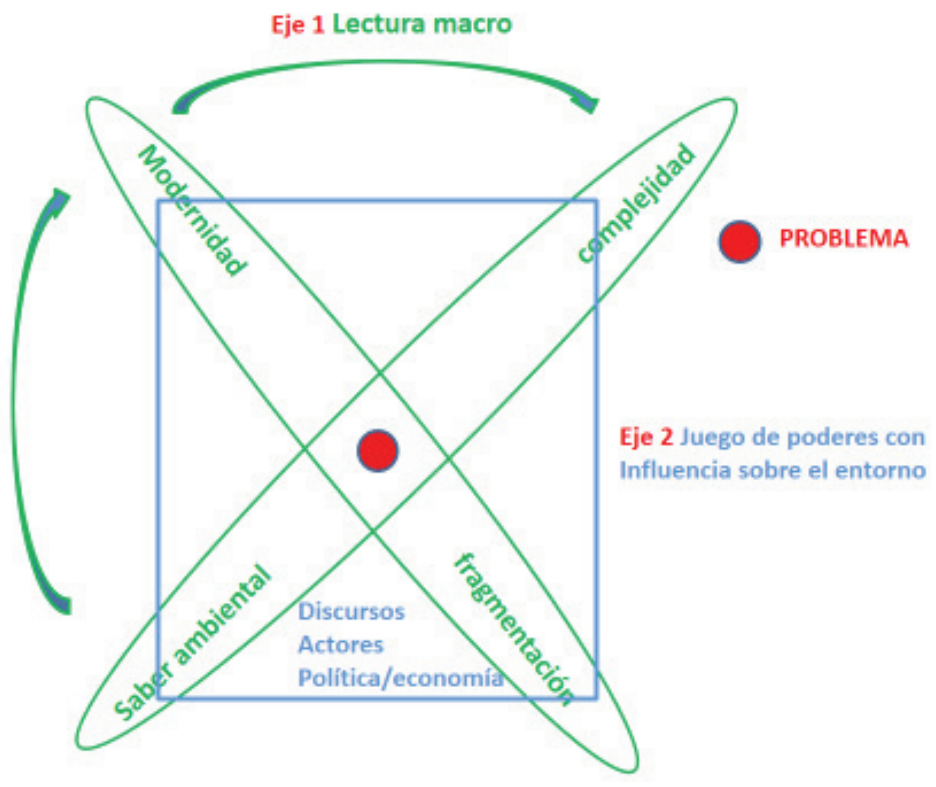


Como se ha dicho ya, el primer eje, es decir, si se ve desde la modernidad o desde la complejidad, influye definitivamente también en la idea de poder. Desde la modernidad, con el peligro de caer aquí en una generalización, el poder se ve en términos de fuerzas, choques, es decir, en términos físicos newtonianos; y desde la complejidad significa pensar desde la vida, y no es posible hablar en términos de jerarquías, sino de rupturas, discontinuidades, bifurcaciones, autoorganización, emergencias, etc. (Maldonado 2013).

Aquí es de señalar a la vez, que en torno al concepto de poder existe un sinfín de reflexiones, desde la filosofía, pasando por la sociología, política y psicología, ya que efectivamente cualquier relación que establece el ser humano con su entorno está mediado por el poder ${ }^{18}$. Por lo tanto, cualquier relación ser humano-naturaleza está relacionada con los diferentes tipos de "juegos" de poder y con sus respectivos intereses, que implican transformaciones concretas sobre el entorno. Las relaciones de poder que se expresan entre los seres humanos conllevan transformaciones del entorno socioambiental, y por esto es necesario identificar algunos componentes para su comprensión. En el presente contexto, de querer entender la relación ser humanonaturaleza, resulta pertinente revisar tres componentes que rodean las relaciones de poder y que están interrelacionados y son interdependientes: primero, la influencia de los discursos socioambientales que influyen

\footnotetext{
18 No es objeto de este trabajo profundizar en teorías sobre el poder; sin embargo, se pueden señalar algunos autores claves, que han reflexionado al respecto en los diferentes campos. Así, a lo largo de la historia, se encuentran autores desde Thomas de Aquino, pasando por Thomas Hobbes, Carlos Marx, Max Weber, Michel Foucault, Hannah Arendt hasta Zygmunt Bauman, y muchos más, quienes han aportado significativamente para comprender los más diversos aspectos del poder desde las más diversas perspectivas e ideologías.
}

en los diferentes sujetos y actores; segundo, las relaciones entre actores y sujetos, y sus formas de organización para ejercer el poder sobre un territorio; y, tercero, la identificación de los diversos intereses políticos y económicos internacionales, nacionales y regionales que están en juego.

Los discursos son un componente clave en la explicación de las relaciones de poder entre los seres humanos, porque describen el mundo de cierta forma y moldean las decisiones humanas que van a transformar el entorno. Existen diferentes formas de comprender el discurso. Una versión puede ser la propuesta por Escobar (1996), al hablar del discurso del desarrollo, en el que plantea claramente cómo el conocimiento, especialmente económico, generado en torno a la idea de desarrollo, se institucionaliza y profesionaliza para poner en práctica esta idea. Por lo tanto, un concepto como el desarrollo sostenible claramente hace parte de este discurso y debe ser contextualizado dentro de éste. Desde la perspectiva de Hajer, el discurso es "un conjunto específico de ideas, conceptos y categorizaciones que son producidas, reproducidas y transformadas en una serie de prácticas, a través de las cuales se da sentido a las realidades física y social" (1995: 44). Aquí Hajer pone el énfasis, por un lado, en conceptos y categorizaciones en las que no se puede olvidar el poder en el saber. En el caso ambiental, sería considerar diferencias entre sostenibilidad y sustentabilidad, economía ambiental o ecológica, y todo el conjunto de ideas que respectivamente subyacen. Por otro lado, Hajer pone el énfasis en las prácticas, a través de las cuales se materializan las diferentes propuestas y políticas ambientales, que comienzan desde las prácticas investigativas y van hasta las prácticas cotidianas de reciclaje. La propuesta de discurso 
de Dryzek (2005) ofrece ya concretamente una clasificación. Él identifica cuatro elementos de análisis (entidades básicas, suposiciones sobre las relaciones naturales, agentes y sus motivaciones, y metáforas centrales) para poder diferenciar nueve discursos ${ }^{19}$ en el ámbito político. Esta lista de los nueve discursos, que no necesariamente es completa, resulta útil e interesante para comenzar a ubicar vertientes, y con ello también intereses económicos, en todo el mar de propuestas políticas.

El análisis de los discursos puede develar el por qué una forma particular de entender el ambiente y los problemas ambientales se vuelve preponderante y con autoridad en un periodo de la historia, frente a otras formas de entender el ambiente que son descartadas (Hajer 1995: 44). El surgimiento de los discursos no se puede desligar del contexto histórico en el que ciertos actores, con sus intereses, compiten por ganar mayor influencia en la sociedad. Por el contrario, los discursos están estrechamente interrelacionados con actores e intereses.

El estudio de los actores y las redes de actores con papeles y capacidades para ejercer poder e incidir en el entorno es un segundo componente a tener en cuenta, pues intervienen las organizaciones no gubernamentales 0 asociaciones civiles, así como también las diversas partes del gobierno, el sector privado y actores en general de la sociedad civil, como periodistas, expertos y académicos, a lo largo de diferentes escalas, desde lo local hasta lo global. Estos actores buscan incidir en el entorno físico y social, por lo tanto, comprender mejor la

Se trata de los siguientes discursos: sobrevivencialista, prometeico, racional administrativo, pragmatismo democrático, racionalismo económico, desarrollo sostenible, modernización ecológica, cambio de conciencia verde y políticas verdes. competencia de los diferentes grupos de interés y sus negociaciones es un factor primordial en el análisis del juego de poderes. Son los actores organizados quienes, de acuerdo con su efectividad para influir a otros actores y a la sociedad, refuerzan o cambian gradualmente los discursos y por lo tanto las formas predominantes de entender las relaciones entre los seres humanos y la naturaleza.

El estudio de los intereses políticos y económicos es el tercer componente que se propone. Lo que está en juego en la actividad política de las sociedades humanas en buena parte es la competencia para permanecer en el poder o para controlar o recibir la asignación de recursos o la defensa de ciertas ideas y posturas sobre el funcionamiento de la sociedad y su relación con el entorno. Los intereses políticos y económicos son un factor determinante en las formas de apropiación de los recursos naturales. Identificar los intereses políticos y económicos de los actores con mayor influencia en la sociedad no se puede desprender del análisis de la forma en que estos actores se organizan y de los discursos en que se legitiman y desde los cuales actúan, ya que es aquí donde a la vez se juegan y se definen los conflictos ambientales. Conflictos que generalmente también están atravesados por y que giran en torno a concepciones divergentes sobre la relación ser humano-naturaleza y sobre el ambiente. Para el abordaje y la comprensión de estos conflictos se ha cristalizado toda un área de estudio que es la ecología política ${ }^{20}$.

Finalmente, no es de olvidar que estas relaciones de poder también influyen sobre el

20 Para profundizar en ecología política, ver autores como Alimonda (2006), Leff (2003), Lipietz (2002). 
uso o no de ciertos marcos teóricos. Así, por ejemplo, los marcos positivistas, modernos, que tienen la habilidad para instrumentalizar y materializar los conocimientos en productos y ganancias acumulables, acorde a su racionalidad economicista, y que otorgaron poder y riqueza a unos cuantos actores y sujetos, claramente propician la reproducción de sus propios modelos, lo cual se ha analizado y demostrado a través de estudios sobre capital cognitivo (Galcerán 2007), geopolíticas del conocimiento (Walsh 2002; 2003), colonialidad del saber (Lander 2000), y otros. Es dentro de estas relaciones de poder que otras formas de conocimiento son marginalizadas, invisibilizadas - exterminadas, a través de diferentes mecanismos, como por ejemplo revistas indexadas o lenguajes normalizados. Toda homogenización, simplificación, parametrización tiende a invisibilizar la complejidad.

Finalmente, para la lectura de la relación ser humano-naturaleza será necesario revisar y considerar también los aspectos culturales y biofísicos, o si se quiere, las bases materiales, como se expone en la siguiente gráfica:

Gráfica 5

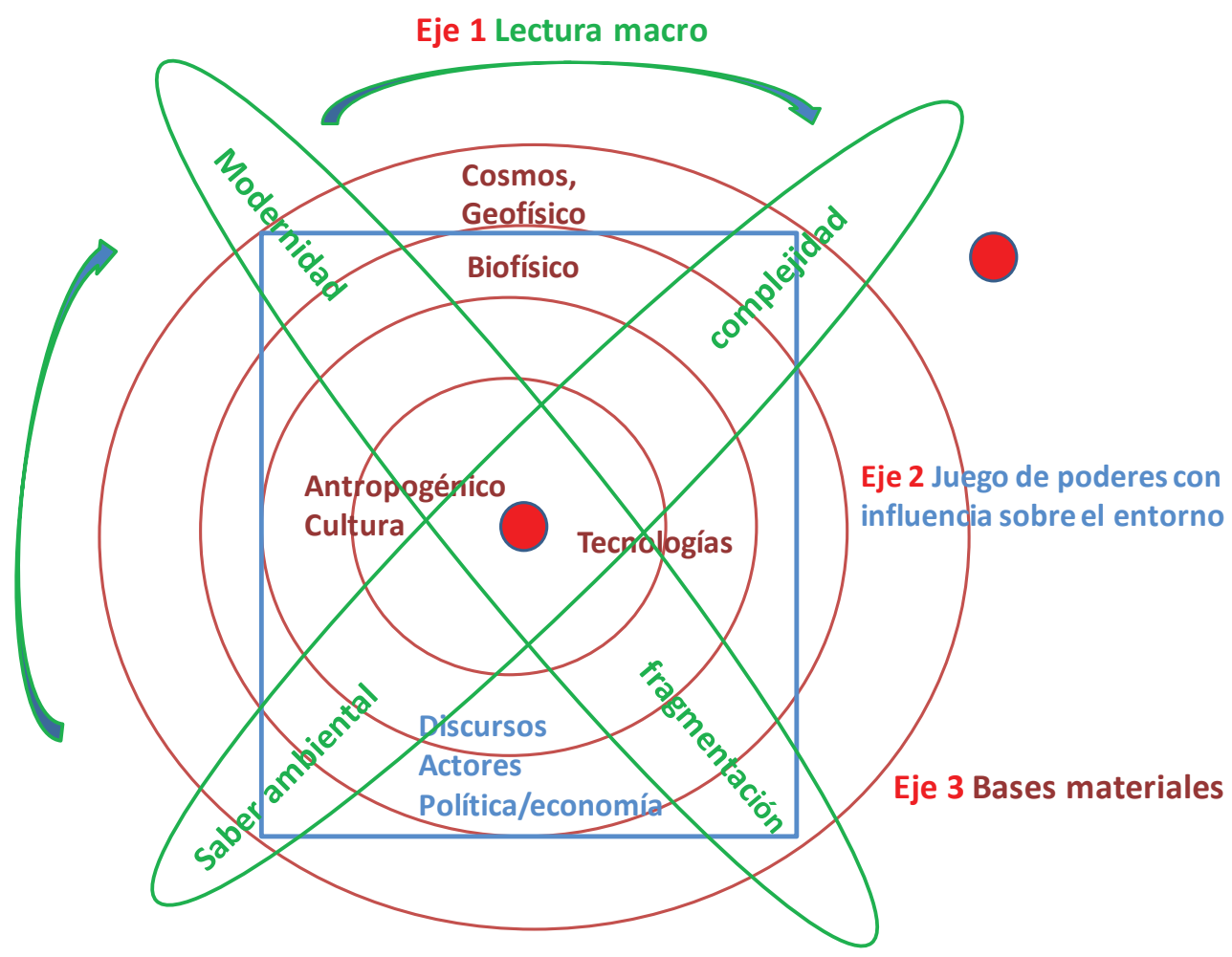


Este tercer eje está representado por círculos que giran en torno al problema central o, si se lee de otra manera, el punto central se encuentra inmerso dentro de estos diferentes círculos, del más al menos amplio: el cosmos, las consideraciones de los ciclos y las condiciones geo y biofísicas, y los aspectos antropogénicos, que se expresan a través de la cultura y las distintas tecnologías que se utilizan. Estos círculos están inspirados en Vidart ${ }^{21}$ y tienen un nivel interesante de complejización, al diferenciar entre procesos básicos, unidades estructurantes, umbrales dinámicos y puentes entre las discontinuidades (1997: 173).

Es necesario hacer énfasis primero en las influencias cósmicas (por ejemplo, intensidad de rayos solares por explosiones solares) y pasar por las condiciones de los ciclos geoquímicos, como del carbono, el fósforo o el nitrógeno, que generalmente son ignorados desde las ciencias sociales $^{22}$. También las condiciones geofísicas resultan indispensables para la toma de decisiones, desde un trazado de carretera hasta la ubicación de viviendas y la determinación de la vocación del territorio en general. En este contexto, entrarían también las corrientes marinas globales y su posible efecto sobre el

Vidart (1997: 143) propone los siguientes círculos:

hilosfera-movimiento universal-“campo unificado" de corpúsculo y onda

Puente I - macromoléculas cristalización - virus

biosfera - fotosíntesis - célula

Puente II - naturaleza humana, dialéctica homínido humánido

antropósfera - comunicación, educación - persona

Puente III - cibernética

tecnosfera - trabajo humano, maquinismo - artefacto

22 Los ciclos geoquímicos como p.ej. del fósforo y nitrógeno son indispensables para posibilitar la vida sobre la tierra, pero estos ciclos están seriamente amenazados por intervenciones antropogénicas. Para ver reflexiones y discusiones al respecto, consultar p.ej.: Liu (2013), De Vires (2012), Barrett (2009), Guinée (2009) y Hoppe (2013). Ver también Tardy (1997) para conocer los ciclos geoquímicos globales a lo largo de la historia de la tierra. clima o comprender la interdependencia entre el Sahara y el Amazonas. Y, finalmente, están las condicionesecosistémicas, es decir, lanecesidad de considerar y comprender lo que significa la biodiversidad, capacidad de resiliencia ${ }^{23}$, procesos entrópicos y neguentrópicos, como flujos de energía y materiales ${ }^{24}$, metabolismo y otros. Estos diferentes aspectos se traducen en la capacidad de reconocer la importancia de la conectividad entre territorios (ver, por ejemplo, actualmente la discusión en torno a la reserva de La Macarena, Colombia (García Márquez, 2012)), zonas de amortiguación ${ }^{25}$ y las posibles amenazas por manipulaciones genéticas. También se requiere de estos conocimientos para comprender la envergadura e importancia de los planes de ordenamiento territorial o de "servicios" ecosistémicos. Todos estos factores no se pueden desconocer para comprender esa interdependencia de la relación ser humano-naturaleza ni para tomar decisiones sociopolíticas y económicas. Es justamente aquí donde se juega la política ambiental (cuyas

23 Uno de los centros más importantes de investigación al respecto es el Stockholm Resilience Centre, Sustainability Science for biosphere Stewardship, http://www.stockholmresilience.org/. Para trabajos latinoamericanos ver p.ej. Cuevas-Reyes (2010)

24 En la economía se vienen realizando propuestas, desde comienzos de los años 70 del siglo pasado con Georgescou-Roegen (1996), para incorporar la entropía en las teorías económicas, y en las últimas tres décadas se viene consolidando con esto el campo de estudio denominado economía ecológica, que se propone entender la economía como un sistema abierto en el cual es clave comprender el flujo de energía y materiales. Esto a diferencia de la economía ambiental, que sigue proponiendo teorías a partir de sistemas cerrados, considerando además como suficiente, la aplicación de las teorías neoclásicas a los "recursos naturales" para que el mercado regule y soluciones los problemas ambientales.

25 Ver toda una iniciativa que gira en torno a este tema: http://www. zonasdeamortiguamiento.org/ en Latinoamérica y ver la dificultad de su manejo (e incomprensión) en el caso de los Parques Naturales concesionados en Colombia, como que se refleja en la siguiente cita "Con esta medida, el Ministerio no busca impedir el ingreso de turistas, sino regularlo y ubicar la infraestructura de alojamiento, distinta a hamacas y carpas, en las zonas de amortiguación" En: http:// www.semana.com/nacion/articulo/parques-naturales-prohibidotocar/335117-3 
decisiones muchas veces se toman por intereses de poder más que basadas en conocimientos de esta envergadura) y el poder en el saber.

Ahorabien, losaspectos culturalesytecnológicos también son diversos y determinantes en el momento de tratar de comprender la relación ser humano-naturaleza. No es lo mismo comprender un lugar en el cual actúan culturas indígenas, rurales o urbanas, si estas se encuentran en el hemisferio norte o sur, en el continente africano, asiático o latinoamericano. En cualquier lugar las culturas han desarrollado formas muy diversas de adaptación a sus medios, así como tecnologías muy específicas, como por ejemplo los sistemas de riego en Perú o en el Himalaya, los de pesca en ríos, lagos o mares, o las tecnologías agrícolas de zonas templadas, tropicales o montañosas, que dependen de las culturas locales. Por lo tanto, si se quiere comprender la relación ser humanonaturaleza será indispensable comprender estas configuraciones y adaptaciones. Desde la antropología se han realizado ya muchos trabajos al respecto (González 1992; Gelles 2002; Doolittle 1995).

\section{Dificultades para la puesta en práctica}

La propuesta hecha hasta aquí está lejos de estar completa, pues es necesario ampliar y profundizar los ejes dos y tres. Sin embargo, puede ser una primera aproximación interesante para comprender las suposiciones de la relación ser humano-naturaleza, que subyacen en las formulaciones y en la ejecución de cualquier proyecto que se denomine ambiental. Se trata de suposiciones que por lo general no se explicitan, pero que en aras de la coherencia tanto interna del proyecto, como con los objetivos deseados (de los diferentes actores), requieren ser discutidos y visibilizados. Este ejercicio puede resultar arduo, difícil y muchas veces obviado, por diversas razones, como por ejemplo falta de tiempo en proyectos financiados, falta de disposición al diálogo en grupos multidisciplinarios, falta de voluntad política, falta de claridad teórica por parte de los responsables, etc.

$\mathrm{Si}$, efectivamente, en un proyecto de investigación se asume este reto, ello requiere, por un lado, de una buena capacidad de abstracción, para ver relaciones y estructuras invisibles, que solo el ser humano es capaz de generar y explicar, como lo son los conceptos, la cosmovisión o las relaciones de poder. Por otro lado, requiere de una buena capacidad de diálogo, así como disposición para lo nuevo y desconocido, para atreverse a caminar por la incertidumbre y el no pragmatismo. En últimas, diría que requiere sobre todo de una "buena química" humana entre los participantes, así como de una gran confianza y respeto, ya que realizar este tipo de ejercicio lleva tanto a entender que hablamos lenguajes disciplinarios diferentes, que se deben comenzar por reconocer y aclarar, como a cuestionamientos disciplinarios profundos, que no deben tomarse personalmente. Es de reconocer aquí también que existen de-formaciones profesionales (disciplinarias) que, a lo largo de la carrera universitaria, han formado personas que piensan, razonan y actúan dentro de unas lógicas muy específicas (compárese por ejemplo un ingeniero, un abogado, un economista, un biólogo o un filósofo; ver gráfica 6) y que en este tipo de ejercicios afloran. Deformaciones que deben ser identificadas para superarlas y poder dialogar. Finalmente, lo que definitivamente es contraproducente para estos procesos son los tiempos y las presiones contractuales, que por lo general están solamente interesados en obtener "resultados concretos". 
Gráfica 6

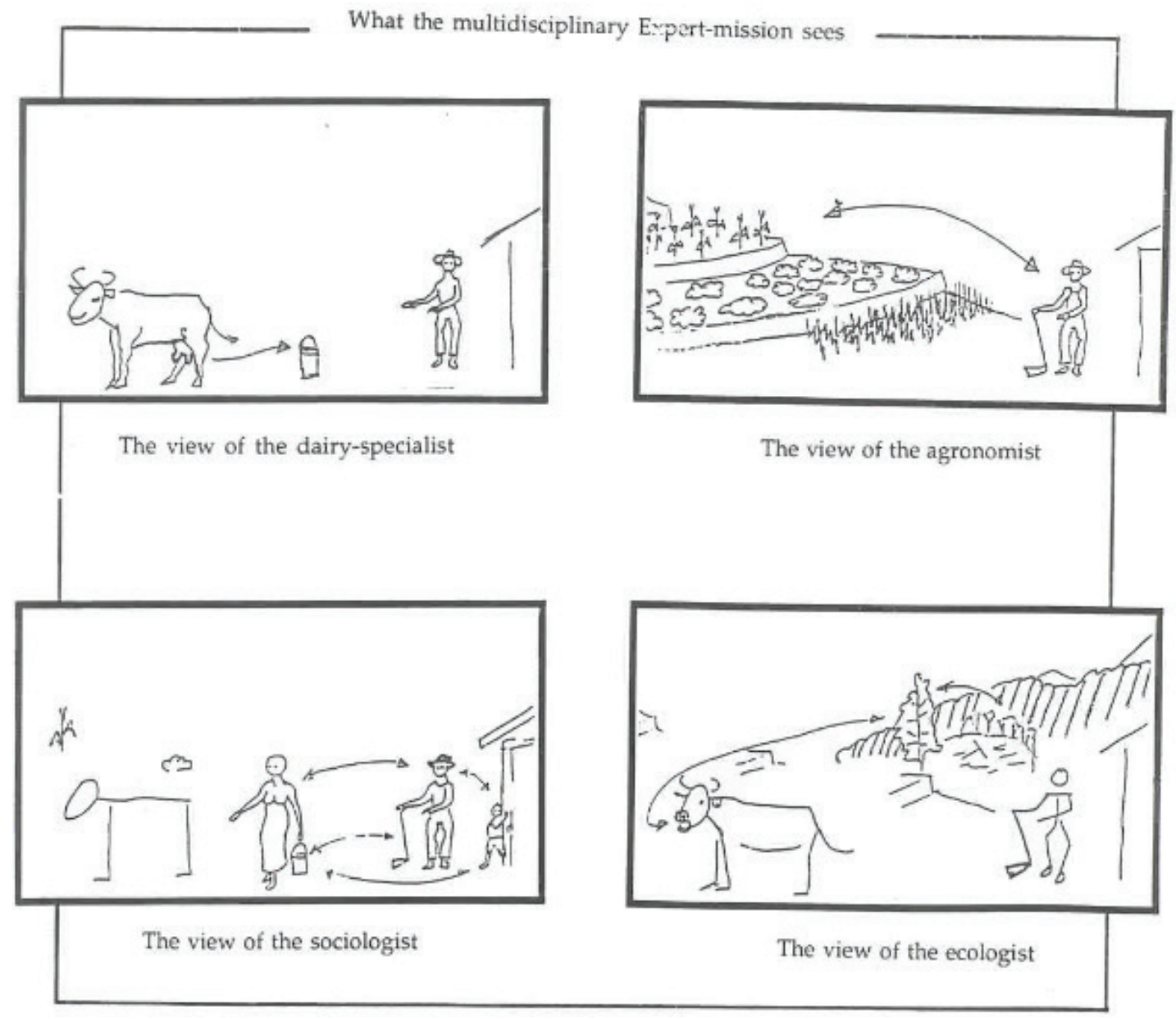

Fuente: Ison 2008: 8

Un obstáculo para semejante reto reside también en que la visión hegemónica del conocimiento, es decir, la ciencia moderna, al estar preocupada por presentar un conocimiento objetivo ha eliminado o invisibilizado al ser humano. Por lo tanto, supuestamente lo único que vale es la descripción "objetiva" del mundo, cuantificación, el muestreo, las estadísticas, etc., que permiten una forma "correcta" de categorizar el mundo y de derivar leyes universales. Esto conlleva muchas veces a pensar que, en lo que respecta a la ciencia, se trata más de aplicarla a través de tecnologías en lugar de comprender los fenómenos. Ello pudiera llevarnos a pensar que no puede ser interpretativa, lo cual lleva a barreras serias de comunicación -que no son 
nuevas, sino históricas- entre las ciencias duras y aplicadas y las ciencias sociales. Son estas las barreras previas a resolver antes de poder hablar de interdisciplinariedad, si se entiende por ello la construcción conjunta de un nuevo objeto de conocimiento, con bases epistemológicas comunes. Esto significa, por un lado, que aún existe una distancia muy grande para lograr proyectos realmente interdisciplinarios. Por el otro, que aún hay camino muy largo por andar para superar esa escisión tan profunda de ser humano-naturaleza en la cosmovisión moderna, al constatar que discusiones en torno a posthumanismo, sentipensar, ontología relacional, aún en los espacios de las ciencias sociales, son marginales y en las ciencias duras y aplicadas casi inexistentes, por no decir desconocidas. Por lo tanto, y dentro del contexto en el que se dan los proyectos de investigación de invervención (mainstream) presionados por el eficientismo y el capitalismo hegemónico-, lograr una coherencia teórica, epistémica, que entienda las implicaciones de las concepciones de naturaleza y la relación ser humano-naturaleza, ya sería un avance importante.

De ahí también surge la convicción de que es hora de recuperar los espacios para la reflexión y el diálogo filosófico, recuperar el sentido de la vida a través de las preguntas por el por qué y el para qué, que han sido desplazados por el pragmatismo del qué y cómo, y a la vez asumir las consecuencias socio-ambientales y sus implicaciones éticas al elegir un marco teórico, filosófico específico.

\section{Bibliografía}

Aguiar Coimbra, J. 2000. Consderações sobre a Interdisciplinaridade, En Philipi, A. et al (eds.). Interdisciplinaridade em Ciências Ambientais. São Paulo: Red de Formação Ambiental, PNUMA, MTC, PADACT, Ciencias Ambientais, Signus Editora.

Alimonda H. (comp.) 2006. Los tormentos de la materia: aportes para una ecología política. Buenos Aires: CLACSO, Disponible en: http://biblioteca.clacso.edu.ar/clacso/gt/20101002065259/ alimonda2.pdf (consultado mayo 2016).

Ángel Maya, A. 2002. El retorno de ĺcaro. La razón de la vida. Muerte y vida de la filosofía Una propuesta ambiental. México: PNUMA.

1996. El reto de la vida. Ecosistema y cultura, una introducción al estudio del medio ambiente. Bogotá: ECOFONDO.

Apostel, L. (coord.) 1979 [1972]. Interdisciplinariedad, problemas de la enseñanza y de la investigación en las universidades. México, D.F.: OECD, ANUIES.

Banco Mundial, Disponible en: http://datos.bancomundial. org/indicador/SP.URB.TOTL.IN.ZS y http://www.bancomundial. org/temas/cities/datos.htm (consultado abril 2015).

Barrett, J. E., Gooseff, M. N., \& Takacs-Vesbach, C. C. 2009. "Spatial variation in soil active-layer geochemistry across hydrologic margins in polar desert ecosystems". Hydrology \& Earth System Sciences, 13(12): 2349-2358.
Berman, M. 2001 [1981]. El reencantamiento del mundo, Santiago de Chile: Cuatro Vientos.

Bertalanffy, v. L. 1989 [1968]. Teoría general de los sistemas. Fundamentos, desarrollo, aplicaciones. México: Fondo de Cultura Económica.

Cuevas-Reyes, P. 2010. "Importancia de la resiliencia biológica como posible indicador del estado de conservación de los ecosistemas: implicaciones en los planes de manejo y conservación de la biodiversidad". Revista de la DES Ciencias Biológico Agropecuarias, Biológicas 12 (1). Disponible en: http://www.biologicas.umich.mx/index.php/biologicas/article/ view/67/67 (consultado mayo 2016).

De Vries, J. W., Groenestein, C. M. \& De Boer, I. M. 2012. "Environmental consequences of processing manure to produce mineral fertilizer and bio-energy". Journal Of Environmental Management 102: 173-183. Disponible en: http://www.ncbi.nIm. nih.gov/pubmed/22459014 (consultado mayo 2016).

Descola, P. 2012 [2005]. Más allá de naturaleza y cultura. Buenos Aires: Amorrortu.

Doolittle W. E. 1995. "Indigenous Development of Mesoamerican Irrigation" Geographical Review, 85 (3): 301-323.

Dryzek, J. 2005 [1997]. The politics of the earth, environmental discourses. Oxford: Oxford University Press. 
Dürr, H.-P. 2009. Warum es ums Ganze geht, Neues Denken für eine Welt im Umbruch. München: Oekom Verlag.

Eschenhagen, M. L. 2016. "Conceptos, teorías y paradigmas: caminos para identificar vertientes del pensamiento ambiental" En Floriani, D. y Elizalde Hevia, A. (orgs.). América Latina: Sociedade e meio ambiente teorias, retóricas e conflitos em desenvolvimento (pp. 43-78). Curitiba: Editora da UFPR.

2009. La educación ambiental superior en América Latina: Retos epistemológicos y curriculares. Bogotá: ECOE, U.D.C.A, Red Colombiana de Formación Ambiental.

Escobar, A. 1996. La invención del Tercer Mundo, construcción y deconstrucción del desarrollo. Bogotá: Norma.

2014. Sentipensar con la tierra. Nuevas lecturas sobre desarrollo, territorio y diferencia. Medellín: Ediciones UNAULA.

Galcerán H. 2007. "Reflexiones sobre la reforma de la Universidad en el capitalismo cognitivo". Revista Nómadas 27: 8697. Disponible en: https://www.ucentral.edu.co/images/editorial/ nomadas/docs/nomadas_7_reflexiones.pdf (consultado mayo 2016).

García Márquez, J. R. 2012. Documento de consultoría para el proyecto AMAZONIA POSIBLE Y SOSTENIBLE, Corredores biológicos en la Amazonia colombiana: Estado actual, amenazas y conectividad, Disponible en: http://www.academia. edu/11120255/Documento_de_consultor\%C3\%ADa_para_el_ proyecto_AMAZONIA_POSIBLE_Y_SOSTENIBLE_Corredores_ biol\%C3\%B3gicos_en_la_Amazonia_colombiana_Estado_ actual_amenazas_y_conectividad_(consultado mayo 2016).

Gelles, P. H. 2002. Agua y poder en la sierra peruana. La historia y política cultural del riego, rito y desarrollo. Lima: Fondo Editorial de la Pontificia Universidad Católica del Perú.

Georgescou-Roegen, N. 1996. La Ley de la Entropía y el proceso económico. Madrid: Fundación.

González Alcantud, J. \& Malpica Cuello, A. (coord.) 1992. El agua, mitos, ritos y realidades. Granada: Antropos.

Guinée, J. B., Heijungs, R., \& van der Voet, E. 2009. "A greenhouse gas indicator for bioenergy: some theoretical issues with practical implications". International Journal Of Life Cycle Assessment 14(4): 328-339.

Hajer, M. 2005[1995] . The Politics of Environmental Discourse. Ecological Modernization and the Policy Process. Oxford: Clarendon Press.

Haraway, D. J. 1991. Simians, cyborgs, and women. The reinvention of nature. Londres: Free Association

1996. Universal donors in a vampire culture. It's all in the family: biological kinship categories in the twentieth-century United States. En Cronon, W. (ed.). Uncommon ground. Rethinking the human place in nature (p. 321-376). Nueva York: W.W. Norton \& Company

Hoppe, R., Wesselink, A., \& Cairns, R. 2013. "Lost in the problem: the role of boundary organisations in the governance of climate change". Wires: Climate Change 4(4): 283-300.

Ingold, T. (2000). The perception of the environment: essays on livelihood, dwelling and skill. London: Routledge.
Ison, R. 2008. "Methodological challenges of trans-disciplinary research: Some systemic reflections". Natures Sciences Sociétés 16(3): 241-251. Disponible en: http://oro.open.ac.uk/17553/1/ Ison_2008.pdf (consultado mayo 2016).

Ison, Ray (2008). Understandings and practices for a complex, coevolutionary systems approach. In Proceedings International Symposium: Selected Topics on Complex Systems engineering applied to Sustainable Animal Production (p. 29-31). Michoacán: Morelia. Disponible en: http://oro.open.ac.uk/27354/2/ understandings.pdf (consultado mayo 2016).

Kohn, E. 2013. How forests think. Toward an Anthropology Beyond the Human, Berkeley: University of California Press.

Kuhn, T. 2000 [1962]. La estructura de las revoluciones científicas. Bogotá: Fondo de Cultura Económica.

Lander, E. 2000. La colonialidad del saber: eurocentrismo y ciencias sociales. Perspectivas latinoamericanas, Buenos Aires: CLACSO.

Leff, E. 2004. Racionalidad ambiental, la reapropiación social de la naturaleza. México: Siglo XXI. Siglo XXI. 2006. Aventuras de la epistemología ambiental, México:

2007. Más Allá de la Interdisciplinariedad. Racionalidad Ambiental y Diálogo de Saberes. Disponible en: http://www.nepam.unicamp.br/doutorado/ (consultado febrero 2007).

s.f. Economía y democracia: Las alternativas para el desarrollo sustentable y equitativo. Disponible en: http://www. ambiente.gov.ar/infotecaea/descargas/leff06.pdf (consultado mayo 2016).

2000. Espacio, lugar y tiempo La reapropiación social de la naturaleza y la construcción local de la racionalidad ambiental Desenvolvimento e Meio Ambiente. Curitiba: Editora da UFPR. Disponible en: http://www.paginaspersonales.unam.mx/ files/1057/Publica_20120909003904.pdf (consultado mayo 2016). 2003. La ecología política en América Latina: un campo en construcción. Sociedade e Estado 18(1-2): 17-40. Disponible en: http://www.scielo.br/scielo.php?script=sci arttext\&pid=S0102-69922003000100003\&lng=en\&tlng=es. \%20 10.1590/S0102-69922003000100003 (consultado mayo 2016).

Lipietz, A. 2002. ¿Qué es la ecología política? La gran transformación del siglo XXI. Santiago: LOM.

Liu, S., \& Chang, N. 2013. "Geochemical impact of aquifer storage and recovery operation on fate and transport of sediment phosphorus in a large shallow lake". Environmental Earth Sciences 68(1): 189-201.

Lizcano, E. 2006. "METÁFORAS QUE NOS PIENSAN: Sobre ciencia, democracia y otras poderosas ficciones" Revista Intersticios. Madrid: Traficantes de Sueños.

Maldonado, C. 2013. Significado e impacto social de las ciencias de la complejidad. Bogotá: Ediciones desde abajo.

2003, "Marco teórico del trabajo en Ciencias de la Complejidad y siete tesis sobre la Complejidad". Revista Colombiana de Filosofía de la Ciencia 4(9): 139-154. Disponible 
en: http://www.redalyc.org/articulo.oa?id=41400904 (consultado mayo 2016).

2005, Termodinámica y complejidad, una introducción para las ciencias sociales y humanas. Bogotá: Universidad Externado de Colombia.

2013. Significado e impacto social de las ciencias de la complejidad, Bogotá: Desde abajo, Disponible en: http:// www.carlosmaldonado.org/articulos/Significado $\% 20$ e $\% 20$ impacto\%20social\%20de\%20las\%20ciencias\%20de\%20la\%20 complejidad.pdf (consultado mayo 2016).

Mardones, J. M. 2006 [1982]. Filosofía de las ciencias humanas y sociales. Materiales para una fundamentación científica, Colombia: Anthropos, Editorial del Hombre.

Maturana H. \& Varela F. 1998. El árbol del conocimiento. Chile: Universal.

Miranda Vera, C. E. 1997. Filosofía y medio ambiente una aproximación teórica, México: Ediciones Taller Abierto.

Nagel, T. 1974. What Is It Like to Be a Bat? The Philosophical Review 83(4): 435-450.

Revista Semana. 2013. "Parques naturales: prohibido tocar", (2 de marzo 2013). Disponible en: http://www.semana.com/ nacion/articulo/parques-naturales-prohibido-tocar/335117-3 (consultado mayo 2016).

Rifkin, J. 2010. La civilización empática, La carrera hacia una ciencia global en un mundo en crisis. Madrid: Paidós.

Schiemann, G. 1996. Was ist Natur? Klassische Texte zur Naturphilosophie. München: Deutscher Taschenbuch Verlag.

Sejenovich, H. (s.f.) Criterios para evaluar la administración del estado en el desarrollo sustentable. Aportes 12. Disponible en: http://www.asociacionag.org.ar/pdfaportes/12/a12_12.pdf (consultado mayo 2016).

Santos, Boaventura de Sousa (comp.). 2004. Conhecimento prudente para uma vida decente. Um discurso sobre as ciências, Cortez, Sao Paulo

Stockholm Resilience Centre, Sustainability Science for biosphere Stewardship. Disponible en: http://www. stockholmresilience.org/ (consultado mayo 2016).

Tardy, Y. 1997. "Geoquímica Global: oscilações climáticas e evolução do meio ambiente desde quatro bilhões de anos". Estudos Avançados, 11(30): 149-173. Disponible em: http:// www.scielo.br/scielo.php?script $=$ sci_arttext\&pid=S010340141997000200011\&lng=en\&tlng=pt.\%2010.1590/S010340141997000200011 (consultado mayo 2016).

Thompson Klein, J. 1990. Interdisciplinarity, history, theory and practice. Detroit: Wayne State University Press.

Vidart, D. 1997 [1986]. Filosofía ambiental, el ambiente como sistema. Colombia: Nueva América.

Wallerstein, I. (coord.) 2001 [1996]. Abrir las Ciencias Sociales. México: Siglo XXI.

Walsh, C. 2003. "Las geopolíticas del conocimiento y colonialidad del poder Entrevista a Walter Mignolo". Polis 4. Disponible en: http://polis.revues.org/7138 (consultado mayo 2016).

Walsh, C., Schiwy, F., Castro-Gómez S. (edit.) 2002. Indisciplinar las ciencias sociales: Geopolíticas del conocimiento y colonialidad del poder. Perspectivas desde lo andino. Quito: Universidad Andina Simón Bolivar, Ed. Abya Yala.

Zonas de Amortiguamiento de las Áreas Naturales Protegidas en Perú y América Latina. Disponible en: http://www. zonasdeamortiguamiento.org/ (consultado mayo 2016). 
